\title{
Fenologia e soma térmica (graus-dia) para a videira 'Isabel' (Vitis labrusca) cultivada no Noroeste do Paraná1
}

\section{Phenology and thermal summation (degree days) for 'Isabel' grape (Vitis labrusca) produced in the Northwest of Parana State}

\author{
Sérgio Ruffo Roberto ${ }^{2 *}$; Alessandro Jefferson Sato ${ }^{3}$; Éverton Allen Brenner ${ }^{3}$; \\ Cristiano Ezequiel dos Santos ${ }^{4}$; Werner Genta ${ }^{5}$
}

\begin{abstract}
Resumo
O trabalho teve como objetivo caracterizar o comportamento fenológico da videira 'Isabel' (Vitis labrusca) cultivada no norte do Estado do Paraná, bem como caracterizar sua exigência térmica em graus-dia. A área experimental foi instalada em uma propriedade comercial pertencente à Vinícola Intervin ${ }^{\circledR}$, localizada no município de Maringá, PR. O vinhedo foi estabelecido em agosto de 2000 e as plantas foram conduzidas no sistema latada no espaçamento de 4,0 m x 1,0 m. As avaliações tiveram início a partir da poda de frutificação realizada no fim do inverno de 2003. O delineamento experimental foi o inteiramente casualizado com 20 repetições, sendo cada parcela constituída por uma única planta. A fenologia das plantas foi avaliada quanto a duração em dias de cada um dos seguintes subperíodos: poda à gema-algodão; poda à brotação; poda ao aparecimento da inflorescência; poda ao florescimento; poda ao início da maturação das bagas e poda à colheita. A exigência térmica da uva 'Isabel' foi calculada empregando-se o somatório de graus-dia desde a poda até a colheita, bem como para cada um dos subperíodos, considerando-se duas temperaturas-base $\left(10^{\circ} \mathrm{C}\right.$ e $\left.12^{\circ} \mathrm{C}\right)$. Pelos resultados obtidos conclui-se que: a duração do ciclo da poda à colheita da videira 'Isabel' no norte do Paraná é de 127 dias, e a sua exigência térmica é de 1.238,20 graus-dias, sendo a temperatura-base de $10^{\circ} \mathrm{C}$ a mais adequada para o cálculo desta demanda.
\end{abstract}

Palavras-chave: Uva, suco, vinho.

\begin{abstract}
The objetive of this research was to characterize the phenological performance of 'Isabel' grape (Vitis labrusca) produced in the northwest of Parana state, as well to characterize its thermal demand in degree-days. The experimental area was established in a commercial vineyard of Vinícola Intervin ${ }^{\circledR}$, Maringá, PR. The vineyard was planted in August of 2000 and the trees were trained in a pergola system, in a $4.0 \times 1.0 \mathrm{~m}$ spacing. The evaluations started from the winter pruning of 2003 . The random design was used as the statistical model with 20 replications and each plot was composed by one tree. The phenology
\end{abstract}

1 Parte do projeto apoiado pela International Foundation for Science, IFS, Suécia; Fundação Araucária e CNPq.

2 Professor Adjunto e Pesquisador do CNPq em Produtividade em Pesquisa. Área de Fruticultura. Universidade Estadual de Londrina. Departamento de Agronomia. Londrina, PR. E-mail: sroberto@uel.br

3 Acadêmico do Curso de Graduação em Agronomia da Universidade Estadual de Londrina e Bolsista de Iniciação Científica $\mathrm{PIBIC/CNPq}$.

4 Aluno regular do Curso de Pós-Graduação em Agronomia, Área de Concentração em Fitotecnia, Universidade Estadual de Londrina e bolsista da CAPES.

5 Eng. Agr. da ANPEF - Associação Norte Paranaense de Estudos em Fruticultura. Consultor em Fruticultura. Marialva, PR.

* Autor para correspondência 
of grapes was evaluated considering the duration in days of each one of the following subperiods: pruning to bud swell, pruning to beggining of shoot growth, pruning to visible cluster, pruning to flowering, pruning to early ripening and pruning to harvest. The thermal demand of 'Isabel' grape was determined using the degree-days summation from the pruning to harvest, as well for each of the subperiods, considering two base-temperatures $\left(10^{\circ} \mathrm{C}\right.$ and $\left.12^{\circ} \mathrm{C}\right)$. It was possible to conclude that: the duration of the 'Isabel' grape (pruning to harvest) in the northwest of Parana is 127 days, and its thermal demand is $1,238.20$ degree-days. The most suitable base-temperature to calculate this demand was $10^{\circ} \mathrm{C}$.

Key words: Grape, juice, wine.

\section{Introdução}

A viticultura é uma atividade de distinta importância econômica em diversos Estados do Brasil. Implantada em uma área de aproximadamente 68.323 ha e com uma produção anual de 1.054 .934 toneladas (REETZ et al., 2004), a viticultura brasileira se concentra nas regiões sul, sudeste e nordeste, sendo o Rio Grande do Sul, São Paulo, Pernambuco, Paraná e Bahia, em ordem decrescente, os principais Estados produtores (ROBERTO, 2000).

Somente no Estado do Paraná, são cerca de 4.300 hectares ocupados pela cultura, sendo a região nortenoroeste responsável por aproximadamente $20 \%$ do total da área cultivada com uvas de mesa no Brasil e $50 \%$ do Estado (POMMER; MAIA, 2003; CORREAA; BOLIANI, 2001; EMATER, 1999). As videiras 'Itália' e 'Rubi' (Vitis vinifera L.) representam juntas mais de $80 \%$ da produção, caracterizando a região como uma grande produtora de uvas finas de mesa (GENTA, 2000).

Diante deste sistema produtivo voltado basicamente para a produção de uvas de mesa, fazse necessário o desenvolvimento de estratégias de produção visando proporcionar uma maior rentabilidade na exploração vitícola na região, ou seja, dinamizar processos para a verticalização da produção, como por exemplo, destinar parte dela para a elaboração de bebidas como suco de uva e vinhos comuns. Face ao grande e crescente volume de produção de uvas de mesa, o que tem acarretado baixa rentabilidade em algumas safras, já é notável entre os produtores da região norte-noroeste do Paraná, aqueles que buscam o processamento da produção visando uma maior rentabilidade da cultura, porém empregando variedades pouco aptas para estes fins.

O aumento do consumo de sucos pela população é um fenômeno mundial. Nos últimos quatro anos, as vendas de sucos prontos para consumo mais que dobraram no país, e estima-se que o crescimento das vendas do segmento de sucos e bebidas à base de frutas deverá ser de $3,4 \%$ entre 2000 e 2005 , sendo que no período anterior o crescimento foi de 3,2\% (RAMIRO, 2003).

Verifica-se também que a comercialização de vinhos tintos comuns tem aumentando ao longo das últimas duas décadas no Brasil. Em 1980, foram comercializados no país 99.646.124 litros de vinho tinto comum, ao passo que em 2001, a comercialização chegou a 176.793.646 litros, o que representa um incremento significativo desta bebida nos últimos onze anos no país (EMBRAPA, 2004).

Diante destes aspectos, o cultivo de videiras destinadas à vinificação e à elaboração de sucos como a 'Isabel' (Vitis labrusca) é uma alternativa para a verticalização da produção de uvas na região noroeste do Paraná. Devido à carência de estudos sobre o assunto no Estado, este trabalho teve por objetivo avaliar o comportamento fenológico da uva 'Isabel', bem como determinar sua exigência térmica em graus-dias na região norte-noroeste do Paraná.

\section{Material e Métodos}

O trabalho foi conduzido em uma propriedade comercial pertencente à Vinícola Intervin $®$, localizada no município de Maringá, PR. O vinhedo experimental da uva 'Isabel' (Vitis labrusca) foi estabelecido em agosto de 2000 e as plantas estão sendo conduzidas no 
sistema latada no espaçamentos de 4,0 m x 1,0 m. Como muitas uvas americanas são normalmente cultivadas de pés-francos, uma vez que apresentam suficiente resistência à filoxera (CAMARGO, 2004), as videiras avaliadas neste trabalho foram desta maneira formadas (RIZZON; MIELE; MENEGUZZO, 2000).

O delineamento empregado foi o inteiramente casualizado com 20 repetições, sendo cada parcela constituída por uma única planta (BOLIANI; PEREIRA, 1996; LEÃO; PEREIRA, 2001). As avaliações tiveram início em setembro de 2003, a partir da poda de frutificação, e se estenderam até a colheita dos frutos.

Para a realização da poda de frutificação, esperou-se pelo início do inchamento das gemas e em seguida, foi aplicado o regulador cianamida hidrogenada a 2,5\% para a quebra da dormência das gemas das videiras, com o objetivo de se obter brotação uniforme das mesmas. A poda de produção foi realizada deixando-se 4 ou 5 gemas por esporão.

Para a avaliação do comportamento fenológico das variedades, foram etiquetados 2 ramos de cada parcela. Foi avaliada nestes ramos, através de observações visuais, a duração em dias dos seguintes estádios a partir da poda de frutificação, segundo a seguinte classificação baseada em Baggiolini (1952), Pedro Júnior et al. (1989) e Baillod e Baggiolini (1993):

a) Gema-algodão: quando $50 \%$ das gemas atingiram o segundo estádio de desenvolvimento da videira, ou seja, quando as escamas se romperam e apareceu a plumagem;

b) Brotação: quando $50 \%$ das gemas atingiram o quarto estádio, ou seja, a saída das folhas;

c) Aparecimento da inflorescência: quando $50 \%$ dos ramos apresentaram inflorescência, onde os cachos eram visíveis, embora rudimentares;

d) Florescimento: quando $50 \%$ das flores se encontraram abertas (florescimento propriamente dito com flores visíveis);

e) Início da maturação das bagas: quando $50 \%$ das bagas mudaram de coloração, isto é, as bagas com coloração avermelhada e o momento que estas iniciaram o amolecimento; f) Colheita: momento em que $100 \%$ das bagas apresentaram coloração intensa, com teor máximo de sólidos solúveis totais.

Foi caracterizada então a duração em dias de cada um dos seguintes subperíodos: poda à gema-algodão; poda à brotação; poda ao aparecimento da inflorescência; poda ao florescimento; poda ao início da maturação das bagas e; poda à colheita (BOLIANI, 1994; GUERREIRO, 1997). A partir destes dados foi construído um diagrama, representando em escala, a duração em dias de cada uma das fases fenológicas da uva 'Isabel', bem como a duração de cada subperíodo.

Para a caracterização das exigências térmicas da cultivar em estudo, foi utilizado o somatório de grausdia (GD) desde a poda até a colheita, bem como para cada um dos subperíodos, empregando dados climáticos da Estação Meteorológica da Universidade Estadual de Maringá, PR, segundo as seguintes equações propostas por Villa Nova et al. (1972):
a. $G D=\left(T_{m}-T_{b}\right)+\left(T_{M}-T_{m}\right) / 2$, para $T_{m}>T_{b}$;
b. $G D=\left(T_{M}-T_{b}\right)^{2} / 2\left(T_{M}-T_{m}\right)$, para $T_{m}<T_{b}$; e
c. $G D=0$, para $T_{M}<T_{b}$.

onde:

$\mathrm{GD}=$ graus-dia;

$\mathrm{T}_{\mathrm{M}}=$ temperatura máxima diária $\left({ }^{\circ} \mathrm{C}\right)$;

$\mathrm{T}_{\mathrm{m}}=$ temperatura mínima diária $\left({ }^{\circ} \mathrm{C}\right) ; \mathrm{e}$

$\mathrm{T}_{\mathrm{b}}=$ temperatura base $\left({ }^{\circ} \mathrm{C}\right)$.

Os graus-dia foram então calculados para duas temperaturas-base $\left(10\right.$ e $\left.12^{\circ} \mathrm{C}\right)$, a fim de se estabelecer o menor desvio padrão em dias, de acordo com a seguinte equação proposta por Arnold (1959):

$$
\mathrm{Sd}=\mathrm{Sdd} / \mathrm{x}_{\mathrm{t}}-\mathrm{t}_{\mathrm{b}}
$$

onde:

$\mathrm{Sd}$ = desvio-padrão em dias;

Sdd = desvio-padrão em graus-dia;

$\mathrm{x}_{\mathrm{t}}=$ temperatura média do ar no período considerado

$\left({ }^{\circ} \mathrm{C}\right)$; e 
$\mathrm{t}_{\mathrm{b}}=$ temperatura-base $\left({ }^{\circ} \mathrm{C}\right)$

\section{Resultados e Discussões}

Na Figura 1 está apresentada a duração em dias dos estágios fenológicos da videira 'Isabel', bem como a duração de seu ciclo na região.

Observa-se que o ciclo dessa videira (poda à colheita) foi de 127 dias, sendo que a duração dos subperíodos poda à gema-algodão, poda à brotação, poda ao aparecimento da inflorescência, poda ao

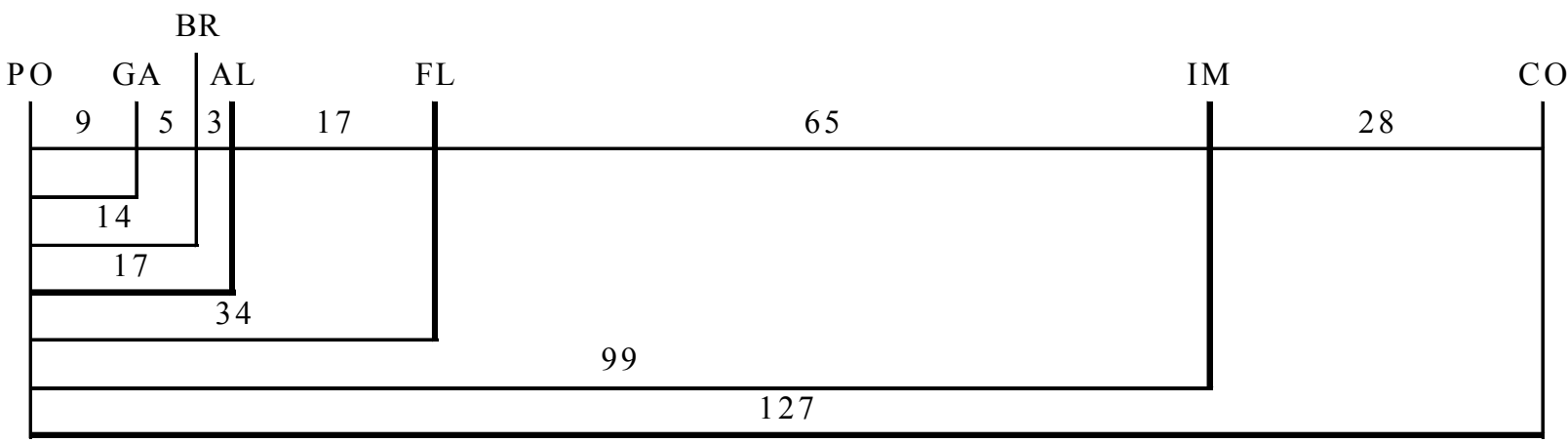

Figura 1. Duração em dias dos estágios fenológicos da videira 'Isabel' (Vitis labrusca) em Maringá, PR. Poda (PO); Gema-Algodão (GA); Brotação (BR); Aparecimento da Inflorescência (AI); Florescimento (FL); Início da Maturação das bagas (IM); Colheita (CO).

florescimento e poda ao início da maturação das bagas, foi de 9, 14, 17, 34 e 99 dias, respectivamente.

Segundo Camargo (2004), a videira 'Isabel' na Serra Gaúcha, RS, apresenta ciclo de 169 dias, ou seja, 42 dias mais longo que em Maringá, enquanto Regina et al. (2003), relatam que o ciclo da videira 'Isabel' no sul de Minas Gerais é de 177 dias, ou seja, 8 dias mais longo que na Serra Gaúcha. Lima et al. (2003) descrevem que o ciclo da videira 'Isabel' cultivada nas condições do Submédio do Vale do São Francisco, BA, é reduzido quando comparado ao da Serra Gaúcha, sendo de 94 dias do período do florescimento à colheita, o que é semelhante ao observado em Maringá, que é de 93 dias para este período (Figura 1). Outros autores, como Maia, Camargo e Nachtigal (2002), relatam que a videira 'Isabel' cultivada em Campina Verde, MG, no sistema de latada, apresenta ciclo de 140 dias, ou seja, 13 dias mais longo que em Maringá.

Estas diferenças entre os ciclos nas diferentes regiões podem ser explicados pelas diferentes condições climáticas existentes, uma vez que Maringá e o Vale do São Francisco, por apresentarem temperaturas médias mais elevadas que a Serra Gaúcha, propiciam desenvolvimento vegetativo superior das videiras, reduzindo assim o seu ciclo.

Os resultados apresentados reforçam as afirmações de Abrahão e Nogueira (1992), que relataram que a avaliação do comportamento fenológico das videiras propicia o conhecimento e a definição das épocas em que ocorrem as diversas fases do período vegetativo das plantas, favorecendo a melhor utilização das práticas culturais, bem como fornece informações ao viticultor para o conhecimento antecipado das prováveis datas de colheita. Devido ao ciclo mais curto desta videira na região, é possível que duas safras anuais possam ser obtidas empregando-se as técnicas utilizadas para a produção de uvas finas de mesa fora de época na região.

Na Tabela 1 encontram-se as exigências térmicas expressas em graus-dia (GD) de cada subperíodo da videira 'Isabel'. 
Tabela 1. Soma térmica, em graus-dia (GD), calculada para temperaturas-base de 10 e $12^{\circ} \mathrm{C}$, e desvio-padrão ( $\mathrm{Sd}$ ) em dias de cada subperíodo para a videira 'Isabel' (Vitis labrusca) em Maringá,'Pß̉abel'

\begin{tabular}{lcccc} 
Subperíodos & \multicolumn{2}{c}{$10^{\circ} \mathrm{C}$} & \multicolumn{2}{c}{$12^{\circ} \mathrm{C}$} \\
\cline { 2 - 5 } & GD & Sd & GD & Sd \\
\hline PO-GA & 54,78 & 0,38 & 45,11 & 0,54 \\
GA-BR & 56,60 & 0,10 & 51,60 & 0,11 \\
BR-AI & 36,15 & 0,07 & 33,15 & 0,08 \\
AI-FL & 156,95 & 0,17 & 139,95 & 0,21 \\
FLO-IM & 645,42 & 0,12 & 580,42 & 0,14 \\
IM-CO & 288,30 & 0,06 & 260,30 & 0,07 \\
\hline PO-CO & $1.238,20$ & 0,16 & $1.110,52$ & 0,19 \\
\hline
\end{tabular}

PO-GA: poda - gema algodão; GA-BR: gema algodão - brotação; BR-AI: brotação - aparecimento da inflorescência; AI-FL: aparecimento da inflorescência - florescimento; FL-IM: florescimento - início da maturação; IM-CO: início da maturação - colheita.

Verificou-se que a temperatura-base mais adequada para os subperíodos e para o ciclo desta videira foi de $10^{\circ} \mathrm{C}$, onde foram obtidos os menores desvios-padrão ( $\mathrm{Sd}$ ) em dias, quando comparada com a temperatura-base de $12^{\circ} \mathrm{C}$. Outros autores determinaram para diversas variedades de videiras no Brasil que a temperatura mais adequada para a caracterização das exigências térmicas do seu ciclo é a de $10^{\circ} \mathrm{C}$ (NAGATA et al., 2000; PEDRO JÚNIOR et al., 1994).

A exigência térmica para a videira 'Isabel' (podacolheita) foi de 1.238,20 GD, considerando-se a temperatura-base de $10^{\circ} \mathrm{C}$, enquanto para os subperíodos: poda à gema-algodão, gema-algodão à brotação, brotação ao aparecimento da inflorescência, aparecimento da inflorescência ao florescimento, florescimento ao início da maturação das bagas e início da maturação das bagas à colheita a exigência foi de 54,$78 ; 56,60 ; 36,15 ; 156,95 ; 645,42$ e 288,30 $\mathrm{GD}$, respectivamente.

De acordo com Mandelli (1984), a exigência térmica da videira 'Isabel' na Serra Gaúcha, RS (poda-colheita), é de 1.393 GD, o que é superior à observada neste trabalho (1.238 GD), o que pode ser explicado pelo fato que o ciclo desta videira na Serra Gaúcha é maior que na região de Maringá, PR
(Figura 1), conforme discutido anteriormente.

Empregando-se os conhecimentos sobre a fenologia e a exigência térmica da uva 'Isabel' no noroeste do Paraná geradas neste trabalho, algumas práticas culturais essenciais poderão ser programadas pelo viticultor, além deste poder também estimar os períodos de maior demanda de mão-de-obra e as prováveis datas de colheitas, em função das épocas de realização da poda de frutificação a cada ano.

Os resultados aqui apresentados, embora propiciem indicações sobre a duração das diferentes fases fenológicas da videira 'Isabel' na região noroeste do Paraná, bem como sobre suas exigências térmicas, devem ser considerados como preliminares, ou seja, observações do comportamento fenológico desta uva destinada à vinificação e à elaboração de sucos na região deverão ser realizados durante vários ciclos produtivos, para que o seu potencial possa ser devidamente avaliado e caracterizado, inclusive para verificar seu potencial para produção intensiva com duas safras anuais, a exemplo do que é realizado tradicionalmente na produção de uvas finas de mesa na região.

\section{Conclusões}

Em função dos resultados obtidos nas condições em que foi realizado o ensaio, foi possível concluir que no primeiro ano de produção da videira 'Isabel' 
no noroeste do Paraná:

a) A duração do seu ciclo da poda à colheita é de 127 dias.

b) A sua exigência térmica, da poda à colheita, é de 1.238,20 GD, sendo que a temperatura-base de $10^{\circ} \mathrm{C}$ a mais adequada para o cálculo desta demanda.

\section{Agradecimentos}

Os autores expressam seus agradecimentos à Vinícola Intervin ${ }^{\circledR}$, International Foundation for Science-IFS, Fundação Araucária, CNPq e ANPEF pelo apoio prestado.

\section{Referências}

ABRAHÃO, E.; NOGUEIRA, D. J. P. Estudo do comportamento fenológico de híbridos franceses $e$ americanos de videiras no sul de Minas. Belo Horizonte: EPAMIG, 1992. (Boletim Técnico, 39)

ARNOLD, C. Y. The determination and significance of the base temperature in a linear heat unit system. Proceedings America Society for Hortculture Science, Virginia, v.74, n.1, p.430-445, 1959.

BAGGIOLINI, M. Les stades repères dans le developpement anual de la vigne. Revue Romande D'Agriculture, de Viticulture et D'Arboriculture, Lausanne, v.8, p.4-6, 1952.

BAILLOD, M.; BAGGIOLINI, M. Les stades repères de la vigne. Revue Suisse de Viticulture, Arboriculture, Horticulture, Nyon, v.25, n.1, p.7-9, 1993.

BOLIANI, A. C. Avaliação fenológica de videira Vitis vinifera L. cv. Itália e cv. Rubi na região oeste do Estado de São Paulo. 1994. Tese (Doutorado em Agronomia) Faculdade de Ciências Agrárias e Veterinárias, Universidade Estadual Paulista, Jaboticabal, 1994.

BOLIANI, A. C.; PEREIRA, F. M. Avaliação fenológica de videiras (Vitis vinifera L.), cvs. Itália e Rubi, submetidas à poda de renovação na região oeste do Estado de São Paulo. Revista Brasileira de Fruticultura, Cruz das Almas, v.18, n.2, p.193-200, 1996.

CAMARGO, U. A. Uvas americanas e hibridas para processamento em clima temperado. Disponível em: $<$ http://www.cnpuv.embrapa.br>. Acesso em: 17 abr. 2004.
CORRÊA, L. S.; BOLIANI, A. C. O cultivo de uvas de mesa no Brasil e no mundo e sua importância econômica. In: SIMPÓSIOBRASILEIROSOBREUVASDEMESA, 1.,2000, Ilha Solteira. Anais...Ilha Solteira: Unesp-Feis, 2001. p.1-19.

EMATER. Diagnóstico preliminar da situação da olericultura e da fruticultura no norte do Paraná. Curitiba, 1999. Projeto Frutinorte

EMBRAPA. Dados da vitivinicultura. Disponível em: $<$ http://www.cnpuv.embrapa.br>. Acesso em: 15 mar. 2004

GENTA, W. A cultura da videira. Marialva: PlantaPlanejamento e Assistência Técnica, 2000.

GUERREIRO, V. M. Avaliação fenológica da videira (Vitis labrusca L. x Vitis vinifera L.) cultivar Niagara Rosada na região de Selvíria-MS. 1997. Dissertação (Mestrado em Agronomia) - Faculdade de Engenharia de Ilha Solteira, Universidade Estadual Paulista, Ilha Solteira, 1997.

LEÃO, P. C. S.; PEREIRA, F. M. Estudo da brotação e da fertilidade de gemas de cultivares de uvas sem sementes nas condições tropicais do vale do submédio do São Francisco. Revista Brasileira de Fruticultura, Cruz das Almas, v.23, n.1, p.30-34, 2001.

LIMA, M. A. C.; LEÃO, P. C. S.; RIBEIRO, A. P. L.; TRINDADE, D. C. G da. Maturação de cultivares de uva nas condições do Submédio São Francisco. In: CONGRESSO BRASILEIRO DE VITICULTURA E ENOLOGIA, 10., 2003, Bento Gonçalves. Anais...Bento Gonçalves: Embrapa Uva e Vinho, 2003. p.196.

MAIA, J. D. G.; CAMARGO, U. A.; NACHTIGAL, J. C. Avaliação da cv. Isabel em três sistemas de condução e em dois porta-enxertos para a produção de suco em região tropical. In: CONGRESSO BRASILEIRO DE FRUTICULTURA, 17., 2002, Belém. Anais... Belém: SBF, 2002. 1 CD-ROM.

MANDELLI, F. Comportamento fenológico das principais cultivares de Vitis vinifera L. para a região de Bento Gonçalves, RS. 1984. Dissertação (Mestrado em Agrometeorologia) - Escola Superior de Agricultura Luiz de Queiroz, Universidade de São Paulo, Piracicaba, 1984.

NAGATA, R. K.; SCARPARE FILHO, J. A.; KLUGE, R. A.; NOVA, N. A. V. Temperatura-base e soma térmica (graus-dia) para videiras 'Brasil' e 'Benitaka'. Revista Brasileira de Fruticultura, Cruz das Almas, v.22, n.3, p.329-333, 2000 .

PEDRO JÚNIOR, M. J.; SENTELHAS, P.C.; POMMER, C. V.; MARTINS, F. P.; GALLO, P. B.; SANTOS, R. R.; BOVI, V.; SABINO, J. C. Caracterização de estádios fenológicos da videira 'Niagara Rosada'. In: CONGRESSO BRASILEIRO DE FRUTICULTURA, 10., 1989, Fortaleza. Anais... Fortaleza: SBF, 1989. v.1, p.453-456. 
PEDRO JÚNIOR, M. J.; SENTELHAS, P. C.; POMMER, C. V.; MARTINS, F. P. Determinação da temperatura-base, graus-dia e índice biometeorológico para a videira 'Niagara Rosada'. Revista Brasileira de Agrometeorologia, Santa Maria,v.2, p.51-56, 1994.

POMMER, C. V.; MAIA, M. L. Introdução. In: POMMER, C. V. (Ed.). Uva: tecnologia da produção, pós-colheita e mercado. Porto Alegre: Cinco Continentes, 2003. p.11-36.

RAMIRO, D. Eles ensinaram o Brasil a beber suco. Veja, São Paulo, v.36, n.2, p.78-79, 2003.

REGINA, M. A. et al. Caracterização agronômica de cinco variedades de videira destinadas à elaboração de sucos de uvas na região de Caldas-MG. In: CONGRESSO BRASILEIRODE VITICULTURA E ENOLOGIA, 10., 2003, Bento Gonçalves. Anais...Bento Gonçalves: Embrapa Uva e Vinho, 2003.p.197.

REETZ, E. et al. Anuário brasileiro da uva e do vinho. Santa Cruz do Sul: Gazeta Santa Cruz, 2004.

RIZZON, L. A.; MIELE, A.; MENEGUZZO, J. Avaliação da uva cv. Isabel para a elaboração de vinho tinto. Ciência e Tecnologia de Alimentos, Campinas, v.20, n.1, p.115$121,2000$.

ROBERTO, S. R. Técnicas de cultivo de uvas de mesa en zonas no-templadas en Brasil. Agricola Vergel, Valencia, n.219, p.151-157, 2000.

VILLA NOVA, N. A.; PEDRO JUNIOR, M. J.; PEREIRA, A. R.; OMETTO, J. C. Estimativa de graus-dia acumulados acima de qualquer temperatura base em função das temperturas máxima e mínima. Ciência da Terra, São Paulo, n.30, p.1-8, 1972 . 
